\title{
Connecting Canadians with neuromuscular disease to research
}

$\mathrm{A}$ scant two years after launching, the Canadian Neuromuscular Disease Registry (CNDR) has data from 1381 registrants and has provided information to researchers on nine occasions. The national patient registry, a collaborative effort by over 40 clinicians and investigators, aims to improve the lives of patients affected by neuromuscular diseases by facilitating research, providing information about clinical trials and improving clinical care through standardization.

The CNDR is recruiting patients across Canada diagnosed with one of the over 40 different neuromuscular diseases, including inherited, inflammatory and degenerative conditions. Registering does not mean automatic recruitment into any particular clinical trial; the onus is always on the patient to contact the study investigators.

Individual neuromuscular diseases are rare, but as a group, they are not uncommon. The overall prevalence of disabling inherited neuromuscular disease presenting in childhood or in later life has been estimated at 1 in 3500 .

The development of the registry was led by Dr. Lawrence Korngut from the University of Calgary in Alberta, Dr. Craig Campbell fromWestern University in London, Ontario, and Megan Johnston from the University of Calgary.

Korngut, the CNDR national principal investigator, stated in an email that patients have responded very positively to the registry. "The opportunity to potentially access information about research studies is highly valued among patients with neuromuscular diseases." While many patients know of registries internationally, they feel more comfortable participating in a registry that is Canadian and complies with Canadian privacy standards, he added.

Shelagh Mikulak, a registrant who has amyotrophic lateral sclerosis (ALS) stated in a registry press release that she joined the registry because it gives her

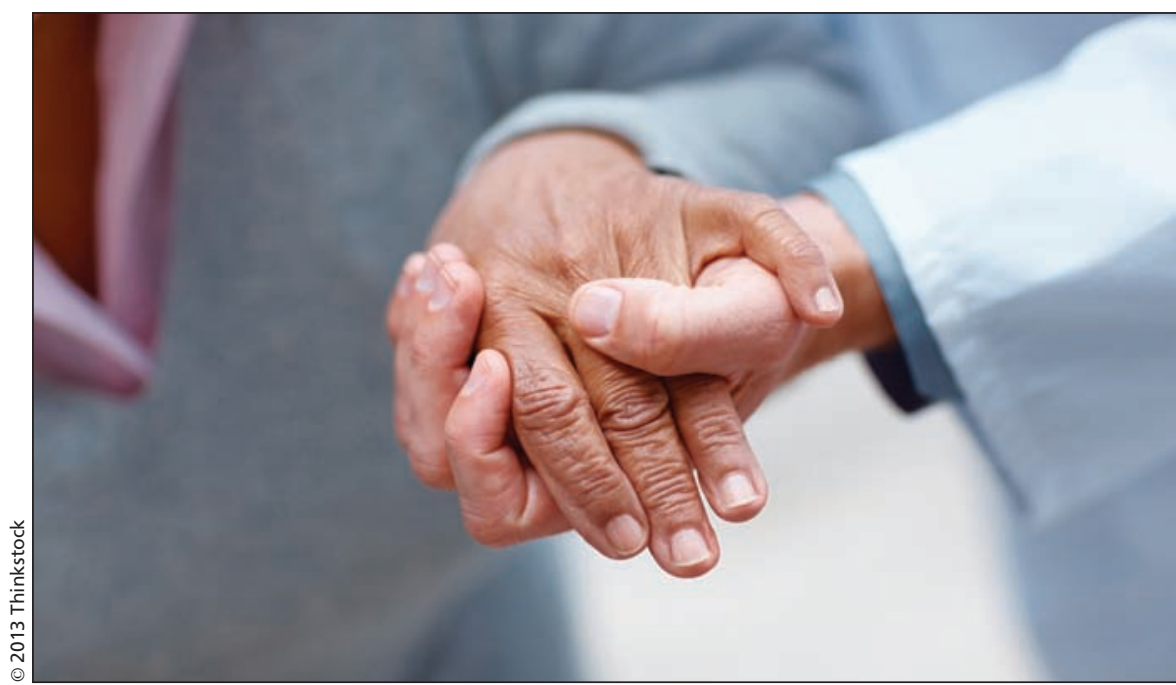

The Canadian Neuromuscular Disease Registry is recruiting patients across Canada diagnosed with one of the over $\mathbf{4 0}$ different neuromuscular diseases.

hope that, "with the information available to researchers there will be significant increase in the number of studies leading to discovering the cause, treatment and cure of ALS."

In an effort to balance the quality and the comprehensiveness of the registry, detailed data are only collected on selected, so-called "index disease" patients who regularly visit a participating clinic. There are currently 691 "index disease" registrants at 20 academic and neuromuscular clinics in six provinces. Clinicians and investigators at four additional sites are preparing to join the registry. If successful, the registry will have participating clinics in eight provinces.

Patients who do not live in cities with affiliated clinics are able to register as "non-index disease" patients by contacting the CNDR national office at the University of Calgary, Alberta. Those in the "non-index disease" category provide only their contact information and confirmation of disease diagnosis. The registry has registrants from 12 of the 13 provinces and territories. The long-term goal is to have clinics in every province and territory.
The registry has been funded through contributions by the ALS Society of Canada, Jesse's Journey, the Families of Spinal Muscular Atrophy Canada Society and the Marigold Foundation. Research projects using data from the registry are independently funded.

Korngut says, "Though early, the CNDR is already facilitating research and is attracting clinical trials to Canada."

Dr. Sanjay Kalra from the University of Alberta in Edmonton, has received funding from the Canadian Institutes of Health Research for a project on magnetic resonance imaging biomarkers in ALS. While the project would have been possible without the existence of the CNDR, Kalra stated in an email that it would have taken "considerable resources to develop the data management infrastructure that is ready and provided by the CNDR." Kalra also credits the existence of the registry with strengthening his team's grant application to the Canadian Institutes of Health Research. — Erin Russell, CMAJ 